\title{
The VEGF and PEDF levels in the follicular fluid of patients co- treated with LETROZOLE and gonadotropins during the stimulation cycle
}

\author{
Jigal Haas ${ }^{1,23^{*}}$, Rawad Bassil ${ }^{1,2}$, Noa Gonen ${ }^{1,2}$, Jim Meriano ${ }^{1,2}$, Andrea Jurisicova ${ }^{1,2}$ and Robert F. Casper ${ }^{1,2}$
}

\begin{abstract}
Background: Previous studies have shown that androgens, in addition to serving as precursors for ovarian estrogen synthesis, also have a fundamental role in primate ovarian follicular development by augmentation of FSH receptor expression on granulosa cells. Recent studies have shown that aromatase inhibitor, letrozole, improves ovarian response to FSH in normal and poor responder patients, possibly by increasing intraovarian androgen levels. Studies in mice also showed an effect of letrozole to increase pigment epithelium-derived factor (PEDF) and to lower vascular epithelial growth factor (VEGF), which might be expected to reduce the risk of ovarian hyperstimulation syndrome (OHSS) with stimulation. The aim of this study was to compare the VEGF and PEDF levels in the follicular fluids of normal responders treated with letrozole and gonadotropins during the ovarian stimulation with patients treated with gonadotropins only.

Methods: A single center, prospective clinical trial. We collected follicular fluid from 26 patients, on a GnRH antagonist protocol, dual triggered with hCG and GnRH agonist. The patients in one group were co-treated with letrozole and gonadotropins during the ovarian stimulation and the patients in the other group were treated with gonadotropins only. VEGF, PEDF, estrogen, progesterone and testosterone levels were measured by ELISA kits.

Results: The age of the patients, the total dose of gonadotropins and the number of oocytes were comparable between the two groups. In the follicular fluid, the estrogen levels ( $2209 \mathrm{nmol} / \mathrm{l} \mathrm{vs} .3280 \mathrm{nmol} / \mathrm{l}, p=0.02)$ were significantly decreased, and the testosterone levels $(246.5 \mathrm{nmol} / \mathrm{l} \mathrm{vs} .40 .7 \mathrm{nmol} / \mathrm{l}, p<0.001)$ were significantly increased in the letrozole group compared to the gonadotropin only group. The progesterone levels $(21.4 \mu \mathrm{mol} / \mathrm{l}$ vs. $17.5 p=\mathrm{NS})$ were comparable between the two groups.

The VEGF levels $(2992 \mathrm{pg} / \mathrm{ml}$ vs. $1812 \mathrm{pg} / \mathrm{ml} \mathrm{p}=0.02)$ were significantly increased and the PEDF levels $(9.7 \mathrm{ng} / \mathrm{ml}$ vs $17.3 \mathrm{ng} / \mathrm{ml} \mathrm{p}<0.001$ ) were significantly decreased in the letrozole group.

Conclusions: Opposite to observations in the mouse, we found that VEGF levels were increased and PEDF levels were decreased in the follicular fluid in patients treated with letrozole during the stimulation cycles. Further investigation is required to determine if patients treated with letrozole during the IVF stimulation protocol are at increased risk for developing OHSS as a result of these findings.
\end{abstract}

Keywords: Letrozole, VEGF. PEDF

\footnotetext{
* Correspondence: jigalh@hotmail.com

'Division of Reproductive Sciences, University of Toronto,

Lunenfeld-Tanenbaum Research Institute, Mount Sinai Hospital, Toronto,

Canada

${ }^{2}$ TRIO fertility partners, 655 Bay St 11th floor, Toronto, ON M5G 2K4, Canada

Full list of author information is available at the end of the article
}

(c) The Author(s). 2018 Open Access This article is distributed under the terms of the Creative Commons Attribution 4.0 International License (http://creativecommons.org/licenses/by/4.0/), which permits unrestricted use, distribution, and reproduction in any medium, provided you give appropriate credit to the original author(s) and the source, provide a link to the Creative Commons license, and indicate if changes were made. The Creative Commons Public Domain Dedication waiver (http://creativecommons.org/publicdomain/zero/1.0/) applies to the data made available in this article, unless otherwise stated. 


\section{Background}

The addition of the aromatase inhibitor, letrozole, to gonadotropin stimulation has been an accepted treatment for oocyte retrieval in women with breast cancer and has been demonstrated to result in lower serum estrogen levels $[1,2]$ an endocrine response that is considered to be favourable in these women who may have estrogen sensitive tumors. In normal women undergoing IVF, lowering serum and follicular estrogen levels could also be potentially beneficial by lowering the risk of ovarian hyperstimulation syndrome (OHSS) since a correlation has been observed between administration of letrozole and lowering the incidence of OHSS [3].

A previous study in a rat model of OHSS [4] demonstrated that treatment with a single dose of letrozole on the hCG trigger day, reduced ovarian diameter, reduced VEGF levels and increased the levels of pigment epithelium derived factor (PEDF). VEGF has been identified as one of the prime causative factors in OHSS while PEDF has been shown to decrease the angiogenic activity of VEGF [5]. These combined results might be expected to reduce the risk of OHSS.

In addition, supraphysiologic levels of estrogen are believed to have a negative effect on oocyte quality [6] and embryo implantation [7] with subsequent adverse pregnancy outcomes [8, 9] and therefore, lowering estrogen levels by adding letrozole could be beneficial in normal women undergoing IVF by increasing implantation and reducing pregnancy complications.

More recently, it has also been observed that breast cancer patients undergoing IVF with the addition of letrozole have an increase in the number of oocytes recovered compared to controls [1, 2]. Several studies have shown that letrozole, improves ovarian response to FSH in normal and poor responder patients, possibly by increasing intraovarian androgen levels (1-3, 11-13). Previous studies have shown that androgens, in addition to serving as precursors for ovarian estrogen synthesis, also have a fundamental role in primate ovarian follicular development by augmentation of FSH receptor expression on granulosa cells [10].

The objective of this study was to measure VEGF and PEDF levels as well as estrogen and testosterone levels in the follicular fluids of normal responder women treated with letrozole and gonadotropins throughout the entire ovarian stimulation for IVF and to compare the results with the follicular fluid levels in patients treated with gonadotropins only.

\section{Methods}

\section{Ethical approval}

The study was approved by the Mount Sinai Hospital ethics review committee, and all couples were required to sign a written informed consent after the provision of complete information.

This study included a total of 26 IVF cycles performed in 26 normal responders treated at our institution between June, 2016 and March, 2017. Patients with PCOS or POR by Bologna criteria were excluded from the study.

The study group included patients on a GnRH antagonist protocol treated with daily letrozole $5 \mathrm{mg}$ together with gonadotropins, from the first day of ovarian stimulation until the trigger day. The control group included patients matched by age, infertility diagnosis and starting gonadotropin dose, on the same GnRH antagonist protocol, treated with gonadotropins only during the stimulation. All the patients included in the study had a dual trigger with hCG and GnRH agonist for final oocyte maturation. The study was not randomized and the decision whether to co-treat with letrozole was made by each treating physician independently.

The decision to administer letrozole was not made for the sake of this study. The treating physicians made this treatment decision independently of the study.

\section{Stimulation protocols}

Gonadotropin treatment (with or without letrozole) was initiated on the 3rd day of menses with the use of recombinant FSH (Gonal F, EMD Serono). Once the leading follicle had reached a size of $13 \mathrm{~mm}$, or E2 levels exceeded $1200 \mathrm{pmol} / \mathrm{L}$, co-treatment with a GnRH antagonist $0.25 \mathrm{mg} /$ day (Orgalutran, Merck) and recombinant LH (Luveris, Serono) or highly purified human menopausal gonadotropin (Menopur, Ferring) was commenced. Follicle growth and hormone levels were serially monitored by ultrasound and blood tests until the dominant follicles reached an average diameter of 18-20 mm. At that point human chorionic gonadotropin (10,000 IU Pregnyl; Merck, Kirkland, Quebec) and GnRH agonist (0.5 mg Suprefact; Sanofi-Aventis, Canada) were administered subcutaneously to trigger ovulation. Thirty-six hours later oocyte retrieval was performed under transvaginal guided ultrasound and needle aspiration.

The follicular fluid (FF) was obtained at the time of oocyte retrieval for the IVF procedure. Only the first follicle from each ovary was collected avoiding blood clots, and only FF from mature full sized follicles $(\geq 16 \mathrm{~mm})$ was included in the study. Only one single follicular fluid sample per patient was included in the study.

After isolation of the oocyte, the clear follicular fluid was centrifuged at $500 \mathrm{~g}$ for $10 \mathrm{~min}$ at room temperature to separate out cellular contents and debris and was then transferred to sterile tubes and frozen at $80{ }^{\circ} \mathrm{C}$ until analysis. The follicular fluid was analyzed for testosterone, estradiol, progesterone by an automated assay (Vitros), and VEGF (Cedarlane; CL76149K) and 
PEDF (Chemicon;CYT 420) concentrations were measured by commercial ELISA using an ELISA kits according to the manufacturer's instructions. We measured follicular fluid progesterone concentrations as a possible way to ensure similar ovarian stimulation in each group.

Comparisons were performed using the paired twotailed student's t-test. A $P<0.05$ was considered statistically significant.

\section{Results}

The age of the patients, the total dose of gonadotropins and the number of oocytes were comparable between the two groups (Table 1). In the follicular fluid, the mean estrogen level (2209 nmol/l vs. $3280 \mathrm{nmol} / \mathrm{l}, p=0.02$ ) was significantly decreased, and the mean testosterone level $(246.5 \mathrm{nmol} / \mathrm{l}$ vs. $40.7 \mathrm{nmol} / \mathrm{l}, p<0.001)$ was significantly increased in the letrozole co-treated group compared to the gonadotropin only group. The mean follicular fluid progesterone level $(21.4 \mu \mathrm{mol} / \mathrm{l}$ vs. $17.5 \mu \mathrm{mol}$, $p=\mathrm{NS})$ was comparable between the two groups (Table 2).

The mean VEGF level $(2992 \mathrm{pg} / \mathrm{ml}$ vs. $1812 \mathrm{pg} / \mathrm{ml}$ $p=0.02)$ was significantly increased and the mean PEDF level $(9.7 \mathrm{ng} / \mathrm{ml}$ vs $17.3 \mathrm{ng} / \mathrm{ml} \mathrm{p}<0.001)$ was significantly decreased in the letrozole group (Table 3).

None of the patients in the study group or in the control group developed early or late OHSS.

\section{Discussion}

In contrast to observations in mice, we found that VEGF levels were increased and PEDF levels were decreased in the follicular fluids of patients treated with letrozole during the stimulation cycles, despite a significant suppression of estradiol concentration in follicular fluid. In the
Table 2 The hormone levels in the follicular fluid from patients co-treated with letrozole and gonadotropins vs. gonadotropins only

\begin{tabular}{llll}
\hline & Letrozole group (13) & Control group (13) & $P$ \\
\hline Estrogen $(\mathrm{nmol} / \mathrm{l})$ & $2009 \pm 1034$ & $3280 \pm 1371$ & 0.01 \\
Testosterone $(\mathrm{nmol} / \mathrm{l})$ & $246.5 \pm 153.2$ & $40.7 \pm 14.3$ & $<0.001$ \\
Progesterone $(\mu \mathrm{mol} / \mathrm{l})$ & $21.4 \pm 8.3$ & $17.5 \pm 10.3$ & 0.3 \\
\hline
\end{tabular}

murine model, letrozole was administered only at the trigger day and not during the ovarian stimulation whereas in our current study, the patients were treated during the entire ovarian stimulation, which might explain the differences between the VEGF and PEDF levels observed.

Similarly to the murine findings, He et al. demonstrated a decrease in the VEGF serum levels after treatment with letrozole in the luteal phase. He found a dose dependent decrease in the levels of VEGF with increasing doses of letrozole administered in the luteal phase [11]. The findings of $\mathrm{He}$ et al. suggested that letrozole could decrease the risk of OHSS although it is not clear if the effect on VEGF and PEDF secretion was a direct action of letrozole or an indirect effect through a reduction in estradiol levels.

A randomized controlled study in hyper-responder patients which aimed to compare the efficacy of letrozole to aspirin during the luteal phase in primary prevention of early ovarian hyperstimulation syndrome showed a lower incidence of OHSS in women receiving letrozole compared with aspirin [3]. In contrast to previous studies, the patients treated with letrozole had higher levels of VEGF in the serum compared to the patients not treated with letrozole. The authors hypothesized that the mechanism of lower incidence of OHSS was independent of

Table 1 Characteristics of the IVF cycles for patients co-treated with letrozole compared to the control group

\begin{tabular}{|c|c|c|c|}
\hline & Letrozole group $(n=13)$ & Without letrozole $(n=13)$ & $P$ value \\
\hline Age (years) & $36.3 \pm 3.9$ & $35.8 \pm 3.7$ & NS \\
\hline AMH (pmol/l) & $14.26 \pm 7.7$ & $16.4 \pm 6.7$ & NS \\
\hline FSH & $7.3 \pm 1.6$ & $6.6 \pm 1.9$ & NS \\
\hline Etiology for infertility & $\begin{array}{l}\text { Unexplained-8 } \\
\text { Male factor-3 } \\
\text { Mechanical-0 } \\
\text { Fertility preservation-2 }\end{array}$ & $\begin{array}{l}\text { Unexplained-7 } \\
\text { Male factor-3 } \\
\text { Mechanical-1 } \\
\text { Fertility preservation- } 2\end{array}$ & NS \\
\hline Length of stimulation (days) & $9.4 \pm 1.8$ & $10.7 \pm 1.7$ & NS \\
\hline Dosage of gonadotropins & $3085 \pm 633$ & $3294 \pm 917$ & NS \\
\hline Oocytes (n) & $11.7 \pm 5.7$ & $12.1 \pm 6.1$ & NS \\
\hline $2 \mathrm{PN}(\mathrm{n})$ & $6.6 \pm 5.1$ & $7.6 \pm 4.4$ & NS \\
\hline Blastocysts (n) & $3.1 \pm 2.2$ & $2.9 \pm 1.9$ & NS \\
\hline Blastocyst rate (blast/2PN) & $46.9 \%$ & $38.1 \%$ & NS \\
\hline E2 levels (pmol/l) & $1032 \pm 375$ & $8069 \pm 3068$ & 0.001 \\
\hline Ongoing Pregnancy rate & 5/11 (45.4\%) & 4/11(36.3\%) & NS \\
\hline
\end{tabular}


Table 3 The VEGF and PEDF levels in the follicular fluid from patients co-treated with letrozole and gonadotropins vs. gonadotropins only

\begin{tabular}{llll}
\hline & Letrozole group (13) & Control group (13) & $\mathrm{p}$ \\
\hline VEGF $(\mathrm{pg} / \mathrm{ml})$ & $2992 \pm 431.7$ & $1812 \pm 462.4$ & 0.02 \\
PEDF $(\mathrm{ng} / \mathrm{ml})$ & $9.7 \pm 5.7$ & $17.3 \pm 8.4$ & $<0.001$ \\
\hline
\end{tabular}

VEGF but rather due to the induction of a luteolytic effect and lower estradiol concentrations which reduced the risk of early-onset OHSS (5).

Although we didn't measure the VEGF or PEDF levels in the serum, we found increased VEGF and PEDF levels in the follicular fluid of letrozole treated patients at the time of oocyte retrieval. In the follicular phase, letrozole reduces serum estrogen levels which results in reduced negative feedback on gonadotrophin secretion from the hypothalamus-pituitary axis [12-14]. By lowering serum estrogen concentrations in the early follicular phase, letrozole causes secretion of more FSH and LH, which acts directly on the granulosa cells and may be responsible for the increased secretion of VEGF. In addition, we found higher intrafollicular levels of testosterone in the letrozole group. We believe that the androgen increase may have a positive effect on follicular development, oocyte maturation and implantation. Since androgens have been shown to increase FSH receptor expression in both murine [15] and primate models [16, 17] it is possible that the increased VEGF level could also be influenced by the impact of increased androgen levels on the granulosa cell responsiveness to FSH in the letrozole treated group.

Previous studies demonstrated $[18,19]$ that women who did not conceive had higher FF VEGF concentrations than women achieving a clinical pregnancy. A negative correlation was observed between FF VEGF concentrations, peak estradiol levels and number of oocytes retrieved. Friedman et al. [20] showed increased VEGF levels in the FF from patients with advanced age compared with younger women. They hypothesized that the higher VEGF concentrations resulted from relative follicular hypoxia which is a stimulant for VEGF production.

As published previously by our group [21], we found a higher number of oocytes, zygotes and blastocysts in women co-treated with letrozole compared to matched patients treated with gonadotropins only. We hypothesize that in normal responders co treated with letrozole the pathophysiology increasing VEGF levels is different and not related to follicular hypoxia. We believe that by lowering serum estrogen concentrations in the early follicular phase, letrozole causes secretion of more FSH and LH, which acts directly on the granulosa cells and may be responsible for the increased secretion of VEGF.
VEGF binds to specific receptors located in endothelial cells called VEGF-R1 (Flt-1) and VEGF-R2. Soluble VEGF-R1 is a naturally produced receptor capable of binding and sequestering VEGF and is able to reduce the level of free, active VEGF [22].

Jakimiuk et al. [23] demonstrated that VEGF/ sFlt-1 ratio in FF on the day of oocyte retrieval in women undergoing IVF procedure, regardless of the type of stimulation protocol, might predict the risk of developing OHSS.

The sFlt-1 contribute to the amount of free, biological active VEGF in FF and later in serum by binding VEGF and thereby depleting the amount of free circulating biological active VEGF.

In our study we didn't measure sFlt-1 levels in the FF and therefore it's still speculative whether free circulating VEGF levels were different between the groups.

Tropea et al. [24], cultured human luteal phase with androgens and demonstrated that different doses of androgens significantly increased VEGF secretion. By culturing the cells with aromatase inhibitor, VEGF levels decreased. We think it's difficult to compare those results with our current study because in our study the patients were treated with letrozole which causes secretion of LH and FSH from the pituitary gland in contrast to the cultured granulosa cells which are not affected by those changes. Another major difference is that the granulosa cells were cultured in the luteal phase whereas in our study the patients were treated with letrozole in the follicular phase and the granulosa cells in the follicular phase may respond differently than in the luteal phase to androgens in terms of VEGF production.

In the letrozole group the estrogen levels in the follicular fluid were significantly lower and more similar to the estrogen levels in the natural cycle compared to estrogen levels in the gonadotropins only group [25]. Previous studies have demonstrated a reduced pregnancy rate with increasing E2/ oocyte ratio $[6,26]$ and therefore we assume that treatment with letrozole which reduces the serum and intrafollicular estrogen concentrations, may have a positive effect on the oocyte quality and embryo development.

Although none of the patients in our study group developed OHSS, we think that further investigation is required to determine if patients treated with letrozole during the IVF stimulation protocol are at increased risk for developing OHSS as a result of our new findings demonstrating increased VEGF levels and decreased PEDF levels after treatment with letrozole.

We conclude that co-treatment with gonadotropins and letrozole during the follicular phase increase the VEGF levels and decrease the PEDF levels in the follicular fluid. Whether co-treatment with letrozole during the follicular phase may increase the incidence of OHSS is still unknown and further studies should be performed to evaluate this risk. 


\section{Abbreviations}

ET: Embryo transfer; FET: Frozen embryo transfer; FSH: Follicle stimulating hormone; IVF: In vitro fertilization; OHSS: Ovarian hyperstimulation;

PEDF: Pigment epithelium-derived factor; VEGF: Vascular epithelial growth factor

\section{Availability of data and materials}

The datasets supporting the conclusions of this article are included within the article.

\section{Authors' contributions}

$\mathrm{JH}$ designed and conducted the study and wrote the initial draft of the manuscript. RB, JM, AJ, NG and RC helped with the study design, data analysis, interpretation and manuscript editing. RB, AJ and NG performed the proteins and hormones measurements. All authors read and approved the final manuscript.

\section{Competing interest}

The authors declare that they have no competing interests.

\section{Ethics approval and consent to participate}

The study was approved by the Research Ethics Board at Mount Sinai Hospital in Toronto.

\section{Consent for publication}

All the patients signed a consent form prior recruited for this study.

\section{Publisher's Note}

Springer Nature remains neutral with regard to jurisdictional claims in published maps and institutional affiliations.

\section{Author details}

'Division of Reproductive Sciences, University of Toronto, Lunenfeld-Tanenbaum Research Institute, Mount Sinai Hospital, Toronto, Canada. ${ }^{2}$ TRIO fertility partners, 655 Bay St 11th floor, Toronto, ON M5G 2K4, Canada. ${ }^{3}$ Department of Obstetrics and Gynecology, Sheba Medical Center, Sackler School of Medicine, Tel-Aviv University, Tel-Hashomer, Israel.

\section{Received: 28 January 2018 Accepted: 9 May 2018}

\section{Published online: 29 May 2018}

\section{References}

1. Pereira N, Hancock K, Cordeiro CN, Lekovich JP, Schattman GL, Rosenwaks Z Comparison of ovarian stimulation response in patients with breast cancer undergoing ovarian stimulation with letrozole and gonadotropins to patients undergoing ovarian stimulation with gonadotropins alone for elective cryopreservation of oocytesdagger. Gynecol Endocrinol. 2016;32:823-6.

2. Quinn MM, Cakmak H, Letourneau JM, Cedars MI, Rosen MP: Response to ovarian stimulation is not impacted by a breast cancer diagnosis. Hum Reprod. 2017:32(3):568-74.

3. Mai Q, Hu X, Yang G, Luo Y, Huang K, Yuan Y, Zhou C. Effect of letrozole on moderate and severe early-onset ovarian hyperstimulation syndrome in high-risk women: a prospective randomized trial. Am J Obstet Gynecol. 2017;216:42 e41-10.

4. Sahin N, Apaydin N, Toz E, Sivrikoz ON, Genc M, Turan GA, Cengiz H, Eskicioglu F. Comparison of the effects of letrozole and cabergoline on vascular permeability, ovarian diameter, ovarian tissue VEGF levels, and blood PEDF levels, in a rat model of ovarian hyperstimulation syndrome. Arch Gynecol Obstet. 2016;293:1101-6.

5. Chuderland D, Ben-Ami I, Kaplan-Kraicer R, Grossman H, Ron-El R, Shalgi R. The role of pigment epithelium-derived factor in the pathophysiology and treatment of ovarian hyperstimulation syndrome in mice. J Clin Endocrinol Metab. 2013;98:E258-66.

6. Orvieto R, Zohav E, Scharf S, Rabinson J, Meltcer S, Anteby EY, Homburg R. The influence of estradiol/follicle and estradiol/oocyte ratios on the outcome of controlled ovarian stimulation for in vitro fertilization. Gynecol Endocrinol. 2007;23:72-5

7. Imudia AN, Goldman RH, Awonuga AO, Wright DL, Styer AK, Toth TL. The impact of supraphysiologic serum estradiol levels on peri-implantation embryo development and early pregnancy outcome following in vitro fertilization cycles. J Assist Reprod Genet. 2014;31:65-71.

8. Ishihara $\mathrm{O}$, Araki $\mathrm{R}$, Kuwahara A, Itakura A, Saito $\mathrm{H}$, Adamson GD, Impact of frozen-thawed single-blastocyst transfer on maternal and neonatal outcome: an analysis of 277,042 single-embryo transfer cycles from 2008 to 2010 in Japan. Fertil Steril. 2014;101:128-33.

9. Wennerholm UB, Henningsen AK, Romundstad LB, Bergh C, Pinborg A, Skjaerven R, Forman J, Gissler M, Nygren KG, Tiitinen A. Perinatal outcomes of children born after frozen-thawed embryo transfer: a Nordic cohort study from the CoNARTaS group. Hum Reprod. 2013;28:2545-53.

10. Weil S, Vendola K, Zhou J, Bondy CA. Androgen and follicle-stimulating hormone interactions in primate ovarian follicle development. J Clin Endocrinol Metab. 1999;84:2951-6.

11. He Q, Liang L, Zhang C, Li H, Ge Z, Wang L, Cui S. Effects of different doses of letrozole on the incidence of early-onset ovarian hyperstimulation syndrome after oocyte retrieval. Syst Biol Reprod Med. 2014;60:355-60.

12. Kamat A, Hinshelwood MM, Murry BA, Mendelson CR. Mechanisms in tissuespecific regulation of estrogen biosynthesis in humans. Trends Endocrinol Metab. 2002;13:122-8.

13. Naftolin F, MacLusky NJ, Leranth CZ, Sakamoto HS, Garcia-Segura LM. The cellular effects of estrogens on neuroendocrine tissues. J Steroid Biochem. 1988;30:195-207.

14. Naftolin F, Romero R. H2-receptor antagonists and sexual differentiation. Gastroenterology. 1984;87:248-9.

15. Laird M, Thomson K, Fenwick M, Mora J, Franks S, Hardy K. Androgen stimulates growth of mouse Preantral follicles in vitro: interaction with follicle-stimulating hormone and with growth factors of the TGFbeta superfamily. Endocrinology. 2017;158:920-35.

16. Gervasio CG, Bernuci MP, Silva-de-Sa MF, Rosa ESAC. The role of androgen hormones in early follicular development. ISRN Obstet Gynecol. 2014;2014:818010.

17. Nielsen ME, Rasmussen IA, Kristensen SG, Christensen ST, Mollgard K, Wreford Andersen E, Byskov AG, Yding Andersen C. In human granulosa cells from small antral follicles, androgen receptor mRNA and androgen levels in follicular fluid correlate with FSH receptor mRNA. Mol Hum Reprod. 2011:17:63-70.

18. Friedman Cl, Seifer DB, Kennard EA, Arbogast L, Alak B, Danforth DR. Elevated level of follicular fluid vascular endothelial growth factor is a marker of diminished pregnancy potential. Fertil Steril. 1998;70:836-9.

19. Ocal P, Aydin S, Cepni I, Idil S, Idil M, Uzun H, Benian A. Follicular fluid concentrations of vascular endothelial growth factor, inhibin a and inhibin B in IVF cycles: are they markers for ovarian response and pregnancy outcome? Eur J Obstet Gynecol Reprod Biol. 2004;115:194-9.

20. Friedman Cl, Danforth DR, Herbosa-Encarnacion C, Arbogast L, Alak BM, Seifer DB. Follicular fluid vascular endothelial growth factor concentrations are elevated in women of advanced reproductive age undergoing ovulation induction. Fertil Steril. 1997:68:607-12.

21. Haas J, Bassil R, Meriano J, Samara N, Barzilay E, Gonen N, Casper RF. Does daily co-administration of letrozole and gonadotropins during ovarian stimulation improve IVF outcome? Reprod Biol Endocrinol. 2017;15:70

22. Sela S, Natanson-Yaron S, Zcharia E, Vlodavsky I, Yagel S, Keshet E. Local retention versus systemic release of soluble VEGF receptor-1 are mediated by heparin-binding and regulated by heparanase. Circ Res. 2011;108:1063-70.

23. Jakimiuk AJ, Nowicka MA, Zagozda M, Koziol K, Lewandowski P, Issat T. High levels of soluble vascular endothelial growth factor receptor $1 /$ sFlt1 and low levels of vascular endothelial growth factor in follicular fluid on the day of oocyte retrieval correlate with ovarian hyperstimulation syndrom regardless of the stimulation protocol. J Physiol Pharmacol. 2017:68:477-84

24. Tropea A, Lanzone A, Tiberi F, Romani F, Catino S, Apa R. Estrogens and androgens affect human luteal cell function. Fertil Steril. 2010;94:2257-63.

25. von Wolff M, Kollmann Z, Fluck CE, Stute P, Marti U, Weiss B, Bersinger NA Gonadotrophin stimulation for in vitro fertilization significantly alters the hormone milieu in follicular fluid: a comparative study between natural cycle IVF and conventional IVF. Hum Reprod. 2014;29:1049-57.

26. Yang JH, Chen HF, Lien YR, Chen SU, Ho HN, Yang YS. Elevated E2: oocyte ratio in women undergoing IVF and tubal ET. Correlation with a decrease in the implantation rate. J Reprod Med. 2001:46:434-8. 